\title{
BACKSCATTER COMMUNICATIONS WITH PASSIVE RECEIVERS: FROM FUNDAMENTALS TO APPLICATIONS
}

\author{
Milutin Stanaćević ${ }^{1}$, Akshay Athalye ${ }^{1}$, Zygmunt J. Haas ${ }^{2,3}$, Samir R. Das ${ }^{4}$, Petar M. Djurić ${ }^{1}$ \\ ${ }^{1}$ Electrical and Computer Engineering, Stony Brook University, Stony Brook, NY 11794, ${ }^{2}$ Computer Science, \\ University of Texas at Dallas, Richardson, TX 75080, ${ }^{3}$ School of Electrical and Computer Engineering, Cornell \\ University, Ithaca, NY 14853, ${ }^{4}$ Computer Science, Stony Brook University, Stony Brook, NY 11794,
}

NOTE: Corresponding author: Milutin Stanaćević, milutin.stanacevic@stonybrook.edu

\begin{abstract}
The principle of backscattering has the potential to enable a full realization of the Internet of Things. This paradigm subsumes massively deployed things that have the capability to communicate directly with each other. Based on the types of excitation and receivers, we discriminate four types of backscattering systems: (i) Dedicated Exciter Active Receiver systems, (ii) Ambient Exciter Active Receiver systems, (iii) Dedicated Exciter Passive Receiver systems, and (iv) Ambient Exciter Passive Receiver systems. In this paper, we present an overview of bacskscattering systems with passive receivers which form the foundation for Backscattering Tag-to-Tag Networks (BTTNs). This is a technology that allows tiny batteryless RF tags attached to various objects to communicate directly with each other and to perform RF-based sensing of the communication link. We present an overview of recent innovations in hardware architectures for backscatter modulation, passive demodulation, and energy harvesting that overcome design challenges for passive tag-to-tag communication. We further describe the challenges in scaling up the architecture from a single link to a distributed network. We provide some examples of application scenarios enabled by BTTNs involving object-to-object communication and inter-object or human-object dynamic interactions. Finally, we discuss key challenges in present-day BTTN technology and future research directions.
\end{abstract}

Keywords - Backscatter-based communication, baterryless tags, Internet of Things, protocols, tag-to-tag networks

\section{INTRODUCTION}

The promise of the Internet of Things (IoT) has fomented research into a wide array of wireless technologies and devices capable of providing the required ubiquitous connectivity at a very large scale. In order to maximize the everywhereness and scalability of the IoT, such devices should satisfy the following two key requirements: (i) very low power consumption allowing for batteryless operation and (ii) direct communication with one another and networking without the need for a central master controller. It is in this context that backscattering technology has seen a rapid emergence in recent years, beyond its traditional uses in radar and more recently in Radio Frequency Identification (RFID). Backscattering is a form of wireless transmission based on modulated reflection of external RF signals. Since the source of the RF signal is external, such transmission does not require an 'active' radio transceiver, allowing devices to function in an extremely low power regime (under $10 \mu W$ ). The power needed to operate the transmitter can be harvested from the external RF signal itself, and thus it is possible for such devices to be batteryless.

While the backscattering transmitter is a necessary constituent of every backscatter system, the type of RF excitation source and the type of receiver can vary, giving rise to different classes of systems and networks. In the broad literature, backscatter systems are classified based on the source of excitation into two types:

1. Dedicated exciter (DE) systems: a source of excitation is deployed specifically for the purpose of enabling backscatter transmissions, and

2. Ambient exciter (AE) systems: backscatter transmissions leverage preexisting sources of excitation in the environment such as TV towers, WiFi APs and cell phone towers.

Independent of the excitation source, we posit that an equally important classifying feature of backscatter systems is the type of receiver. Based on this, we identify the following two subclasses of backscatter systems:

1. Active receiver (AR) systems: the receiver is a device with an on-board radio transceiver capable of IQ demodulation and carrier cancellation resulting, typically, in a very high sensitivity (down to $-110 \mathrm{dBm}$ for data rate of $20 \mathrm{~kb} / \mathrm{s}$ and $30 \mathrm{~mW}$ power consumption for commercially available transceivers [1]), and

2. Passive receiver $(\mathrm{PR})$ systems: the receiver is a radio-less passive device using an envelope detector for signal demodulation resulting in a much lower sensitivity $(-56 \mathrm{dBm}$ for data rate of $8 \mathrm{~kb} / \mathrm{s}$ 
and $236 \mathrm{nW}$ power consumption as in a Bluetooth wakeup receiver [2]).

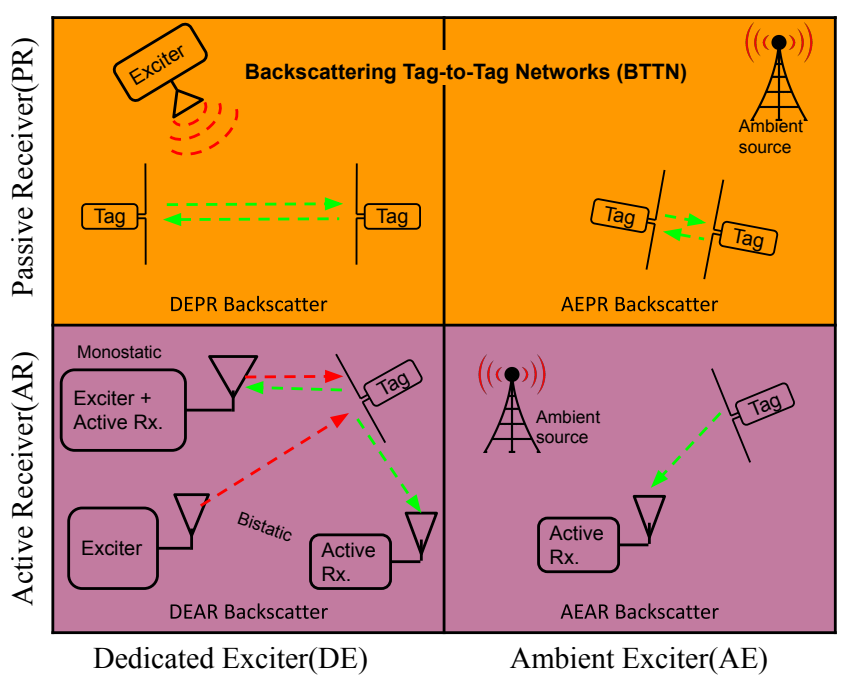

Fig. 1 - Classification of backscatter systems. All such systems contain a backscattering transmitter (Tag). They are classified based on the source of excitation signal and the type of receiver employed.

Combining the above criteria, we classify all backscatter systems into four types as follows:

(i) Dedicated Exciter Active Receiver (DEAR) [3],

(ii) Ambient Exciter Active Receiver (AEAR) [4, 5, 6, 7],

(iii) Dedicated Exciter Passive Receiver (DEPR) [8, 9, $10]$, and

(iv) Ambient Exciter Passive Receiver (AEPR) [11].

This classification is shown in Fig. 1. As pointed out in [12], DEAR systems can be either monostatic where the exciter and receiver functions are on the same device (e.g., traditional RFID) or bistatic with these functions being on different devices.

We focus specifically on passive receiver (PR) backscatter systems which form the basis of the so-called Backscattering Tag-to-Tag Networks or (BTTNs). PR backscatter systems present fundamentally different challenges than AR backscatter systems. The challenges stem from the need to passively receive the backscatter signals in the presence of the interfering excitation with only an envelope detector. However, if these challenges are overcome, then BTTN can fundamentally transform the capabilities of the IoT by enabling all passively tagged "things" to talk directly with each other without any central active controller or master. In AR systems whether of DEAR or AEAR variety, this capability is impractical since the high cost and high powerrequirement of active receivers means that they cannot be used as devices to ubiquitously tag "things" on a large scale.

In summary, the BTTN paradigm utilizing DE or AE can provide a common medium or language for direct peer-to-peer communication between all constituent objects of the IoT irrespective of cost, volume or density of object population. In a sense, the excitation source in BTTN can be thought of as simply illuminating an area, and the tags can "see" each other in this "light." Whether the illumination comes from a natural source (AE approach) or a light bulb (DE approach), the communication between the tags is unaltered.

\subsection{Scalability and practical implications of the AE approach}

Generally, AE backscatter systems are considered to be extremely scalable because they can theoretically enable communication without any deployment. However, there are important practical considerations that can adversely affect this in a real-world IoT deployment.

- AEAR systems: There are widespread efforts in the literature on these systems. The systems are based on the idea of building passive tags that can use ambient excitation to synthesize backscatter packets that are compatible with commodity standards such as WiFi, Bluetooth or ZigBee $[4,5,7]$. A corresponding commodity receiver can then receive this signal and communicate with the tag. This approach significantly complicates the transmit circuitry on the tag. Further, such tags can only be built to synthesize one kind of backscatter packets using one kind of excitation signal. Under this approach, the tags cannot communicate with each other.

- AEPR systems: These systems fall under the BTTN umbrella. While this, in theory, enables maximum scalability, it is important to note the ambient power level requirement. As shown in [13], in order to enable a practical link distance, the required power level is of the order of $-25 \mathrm{dBm}$. Most ambient excitation signals in general indoor environments from various sources including TV towers, cell phone towers or WiFi APs are far below this value.

Thus, a practical implementation of BTTN might invariably call for a dedicated exciter. However, such an exciter is simply an autonomous, RF transmitter that is not part of the communication network and does not centralize the tag-to-tag communications (refer to the light bulb analogy above). Furthermore, the BTTN backscatter modulator is designed for tag-to-tag communication as opposed to tag to commodity receiver communication. This keeps the "language" and thereby the design of the transmitter much simpler. Unlike the 
AEAR approach where backscattering is tuned to one format of the excitation signal, BTTN tags can talk to each other irrespective of the excitation signal format whether it is a CW or a modulated signal. The only requirement is that in the case of a modulated exciter signal, the bandwidth of the tag-to-tag backscatter is lower than the bandwidth of the excitation signal.

Most of the review papers in the literature on the passive backscatter communication focus on the backscatter systems with active receiver(AR) [14] or group both the $\mathrm{AR}$ and PR systems in the same category [12] without addressing specific issues that exist in PR systems. In enabling a single BTTN link and further scaling up to a larger network, a vast array of challenges needs to be overcome. These stem mainly from having to process received signals and mitigate interference in passive receivers, to operate in an extreme low power regime (e.g., [15]), and to communicate in inherently high volume and high density networks. In the rest of this paper, we provide an overview of recent advances in BTTNs, challenges, applications of BTTNs, and future directions for research.

\section{THE FUNDAMENTALS}

One of the most challenging requirements of a $\mathrm{PR}$ is to receive the inherently weak backscatter signal in the presence of a much stronger interfering excitation without IQ demodulation or carrier cancellation capability. We illustrate this challenge with a basic BTTN link consisting of two tags in an area that sees a sufficient level of excitation signal whether DE or AE. In this basic link, at any given time, one of the tags transmits ( $\mathrm{Tx}$ ) and the other one receives $(\mathrm{Rx})$. All BTTN tags are identical, and they switch between Tx and Rx roles based on the MAC-layer and network-layer protocols.

The Tx tag generates the modulated backscatter signal by altering the antenna's reflection cross section. The signal seen at the Rx tag is a superposition of the excitation signal and the modulated backscatter signal from the Tx tag. In the absence of an on-board radio transceiver, the Rx tag has to demodulate the backscatter signal using envelope detection. The received signal has a very low modulation index due to the small amplitude of the backscatter signal combined with the much larger magnitude of the exciter signal. Additionally, as the two signals combine at the Rx tag, the modulation index is significantly impacted by the relative phase difference between the excitation signal and the backscatter signal.

In Fig. 2, we see the characteristic of the received baseband backscatter signal amplitudes as a function of the Tx to Rx distance for an ideal link and a PR backscatter link which constitutes BTTNs. In the PR BTTN link, the relative phase difference between the received

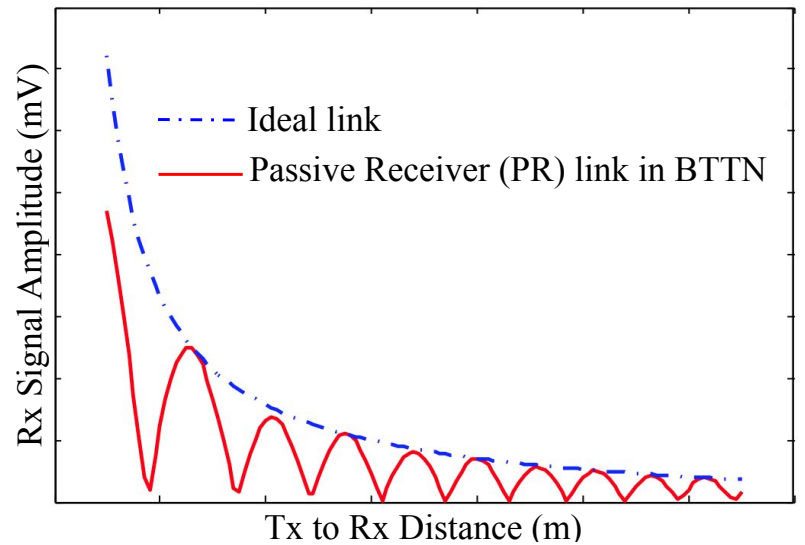

Fig. 2 - Characteristic of backscatter signal amplitude with increasing Tx to $\mathrm{Rx}$ distance in BTTN (PR) link compared to an ideal link without phase cancellation.

backscatter signal and the excitation signal causes a phenomenon referred to as phase cancellation. This is seen in Fig. 2 (the red solid line), where the received signal instead of monotonically decreasing with distance undergoes alternating peaks and nulls, with decreasing peak values $[16,17]$. We note that this phenomenon will also occur in so-called bistatic AR systems [12] where the exciter and receiver are separate. On the other hand, in monostatic AR systems like traditional RFID, the receiver (reader) is able to cancel out the excitation signal and use IQ demodulation for the received backscatter. In this case the received signal amplitude decreases monotonically with distance (the blue dash-dot line). Phase cancellation and low modulation index are two of the most fundamental challenges in enabling basic communication in a PR BTTN link. The phase cancellation can be addressed using a multi-phase backscatter modulator, while signals with low modulation indices are processed with demodulators with innovative architectures $[17,16]$. We note that in a link with AR, there are alternative ways to avoid phase cancellation - by providing a frequency shift to the backscattered signal, thereby avoiding interference with the excitation signal altogether [14].

The maximum communication range of a BTTN link (Tx to Rx distance) depends critically on the excitation power available at the Tx tag regardless of whether the excitation source is DE or AE. Distances up to about $3 \mathrm{~m}$ have been reported with $-20 \mathrm{dBm}$ power available at the Tx tag (5 kbps, BER below $10^{-3}$ ) [16]. Further improvement is possible using coding techniques such as CDMA, but at the expense of data rate. For example, [18] has reported distance up to about $10 \mathrm{~m}$ with similar power levels but providing much slower bit rates, in the order of $100 \mathrm{bps}$. Innovations in the demodulator design can improve the distance and/or improve the data rate. Other innovations are also possible including multiple antennas on the tag [18] or beamforming using multiple tags via a collaborative arrangement. The con- 


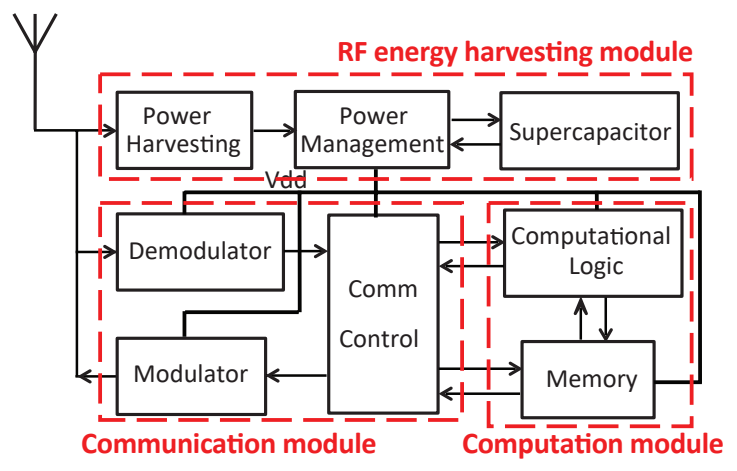

Fig. 3 - The architecture of a backscattering RF tag.

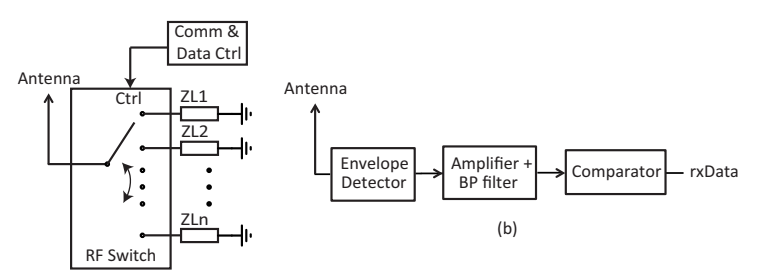

(a)

Fig. 4 - Circuit implementation of (a) modulator and (b) demodulator backscattering-based RF tag.

tributions to the backscatter PR tag-to-tag (T2T) links are summarized in Table 1.

\section{TAG HARDWARE}

The overall architecture of the BTTN tag is shown in Fig. 3. It has three modules, a communication module, an energy harvesting module, and a computation module. The tag optionally interfaces to an external near-zero power sensor. While the sensing and computational module greatly depend on the application, the energy harvesting and communication modules are similar across a wide range of BTTN tags and will be described in greater detail. The control logic manages the operation of the tag while the computational logic, based on the collected data, deduces information on the tag's environment. The power consumption of the BTTN tag is on the order of a few $\mu \mathrm{W}$ as the operating frequency typically does not need to exceed $1 \mathrm{MHz}$ due to a $10 \mathrm{~s}$ of kbps data rate in a tag-to-tag communication link. The critical resource that requires careful optimization on the system level is memory, both volatile and nonvolatile.

\subsection{Communication Module}

The communication module of the BTTN tag incorporates the passive backscattering transmitter and the PR. These operations are implemented, respectively, by the modulator and demodulator sections.

\subsubsection{Modulator architecture}

The modulator of the BTTN tag generates the backscatter signal by varying the impedance of the tag an- tenna between different values (or states). This in turn changes the amplitude and/or the phase of the reflected signal in accordance with the data to be transmitted. This is the conventional backscatter modulation process. In a monostatic AR backscattering system like standard RFID, the impedance is typically varied between two values selected so as to maximize the modulation index of the received signal at the reader in the two states. In a BTTN link on the other hand, the backscatter modulation index depends on the relative phase difference between the exciter signal and the backscatter signal seen at the Rx tag. In order to overcome this problem, it was proposed in $[8,17]$ that the backscatter modulator has the ability to introduce a variable phase offset into the backscattered signal. At some value of the phase offset, the backscatter signal and the excitation signal will be in phase at the $\mathrm{Rx}$ tag resulting in the maximum received backscatter amplitude. When the phase offset is shifted by $\pi / 2$ from this value, the received backscatter amplitude is minimum. The variable phase offset is achieved by switching the tag antenna impedance between a range of systematically designed values; each such impedance corresponds to one phase in a set of phases that span the range from $-\pi / 2$ to $\pi / 2$, as illustrated in Fig. 4(a). The number of different phases is a trade-off between the achieved voltage difference in the received signal, communication data rate and the tag form factor.

\subsubsection{Demodulator architecture}

Demodulating the weak backscatter signal is a fundamental challenge in PR backscatter systems because in the absence of an active radio, the tags need to rely on a passive envelope detector for demodulation. The Rx tag must resolve a weak backscatter signal from the presence of a much stronger external excitation signal resulting in a low modulation index input signal to the demodulator. The communication distance of the BTTN link is directly related to the modulation index that a demodulator can resolve [13]. The demodulator uses an envelope detector that serves as an analog front-end for extraction of the baseband signal. For the detection and demodulation, this analog front-end is followed by a comparator. Because of the much smaller modulation index in the received signal, using conventional RFID tag demodulator architecture leads to short distances of communication [9]. By inserting an amplifier with high-pass filtering after the envelope detection, as illustrated in Fig. 4(b), a tailored demodulator for a tagto-tag link can demodulate signals with a modulation index as low as $0.5 \%$ [13]. The sensitivity of this architecture is related to the power consumption of the amplifier. The ripple voltage in the baseband signal is a critical parameter that determines the performance of the demodulator. To reduce the ripple voltage, higherorder adaptable low-pass filtering could be integrated in the envelope detector prior to signal amplification at a 
Table 1 - Summary of the contributions to backscatter PR tag-to-tag (T2T) links

\begin{tabular}{|l|c|c|c|}
\hline Article & Key contribution & Frequency & Experimental Results \\
& & & \\
\hline \hline$[9]$ & T2T communication concept & $915 \mathrm{MHz}$ & T2T link at $10 \mathrm{~cm}$ \\
\hline$[11]$ & T2T link with ambient exciter & $539 \mathrm{MHz}$ & $1 \mathrm{kbps}$ at $0.75 \mathrm{~m}$ and $-8 \mathrm{dBm}$ \\
\hline$[18]$ & coding technique to extend link distance & $915 \mathrm{MHz}$ & $0.003 \mathrm{kbps}$ at $6 \mathrm{~m}$ and $-20 \mathrm{dBm}$ \\
& multi-antenna tag for increased data rate & $539 \mathrm{MHz}$ & $1000 \mathrm{kbps}$ at $2.1 \mathrm{~m}$ and $-20 \mathrm{dBm}$ \\
\hline$[17]$ & phase cancellation in T2T link & $915 \mathrm{MHz}$ & \\
\hline$[13]$ & theoretical analysis of T2T link & $915 \mathrm{MHz}$ & \\
\hline$[16]$ & demonstration of multi-hop network & $915 \mathrm{MHz}$ & $5 \mathrm{kbps}$ at $3 \mathrm{~m}$ and $-20 \mathrm{dBm}$ \\
\hline$[19]$ & M-PSK for increased data rate & $539 \mathrm{MHz}$ & $20 \mathrm{kbps}$ at $0.75 \mathrm{~m}$ \\
\hline$[10]$ & MAC protocol & $915 \mathrm{MHz}$ & multi-hop T2T links reach $5.65 \mathrm{~m}$ \\
\hline
\end{tabular}

cost of chip area and power consumption.

\subsection{Energy harvesting architecture}

The RF energy harvesting module acquires energy from the external excitation signal. A power harvesting circuit comprises rectification of the incident $\mathrm{AC}$ voltage, followed by multiplication and regulation that provides stable DC supply voltage for the operation of the tag. The energy efficiency of the conventional power harvesting circuit is optimized for a certain range of input power. As the input power can exceed the power consumption of the tag, the extra energy can be stored using a supercapacitor. This enables the operation of the tag when the harvested energy is lower than the instantaneous power consumption. The size of the supercapacitor is limited by the form factor of the tag. The power management logic optimizes the charging of the supercapacitor based on the incident RF power and the power needs of the tag operation.

Based on the incident RF power, the stored energy and the operation of the tag, e.g., backscatter, receive or compute, the power management module directs the tag's operation. The operation of such tags powered by RF harvested energy and low capacity supercapacitors introduces some unique challenges compared to those of a traditional sensor node. Sensor nodes incorporate active radios that dominate the power budget. Though significant steps have been made in reducing their power consumption at the receiver end [1], the transmit power still dominates the operation as the radios must generate the RF carrier signal used for communication. The principal difference between the power budgets of conventional sensor nodes and the RF tags is that the tags operate at orders of magnitude of lower power consumption due to the low energy cost of the communication, as the energy cost of their communication can be orders of magnitude lower than for the nodes comprising active radios. This is because tags only reflect (backscatter) externally supplied RF signals and do not generate any signal on their own. However, in BTTN tags there is no such dominance - the energy costs for communica- tion between the tags and computation are of the same order of magnitude. Further, the different energy costs for performing different operations on the tags lead to a unique power management paradigm for BTTN.

\section{SCALING FROM A SINGLE LINK TO A FULL NETWORK: ROUT- ING FOR IOT APPLICATIONS}

\subsection{From a Link to a Network}

Extending a single link communication to a tag network is far from trivial [20]. Two issues need to be considered: topology formation and routing. The topology formation involves selection of network links for communication based on the energy states of the individual tags. This decision typically involves tags beyond local neighborhoods and may require dynamic operation as the tags' energy states continuously vary.

For communication across a tag-to-tag link, there must be enough $\mathrm{RF}$ power reaching the $\mathrm{Rx}$ tag to power up the receiving tag for effective demodulation and then to do any needed post-demodulation computation (e.g., MAC, routing decisions). The power needed for effective demodulation is dependent on the modulation index, which in turn depends on the wireless channel conditions that determine the powers reaching the tags. This is heavily influenced by the tag and exciter locations. As mentioned in Section 3.2, the energy management module decides the power split among the various operations. The Rx tag in a weak link may have to decide whether to receive a packet at all if it may not be able to forward it immediately for a lack of enough available power. Similarly, a more "energy-rich" tag may be able to take up more responsibilities for routing or MAC protocol decisions.

\subsection{Routing and MAC}

The challenges of designing routing and MAC protocols for BTTN arise from the unique characteristics of the backscattering environment, including the extreme lowpower operation and from the intended BTTN applica- 
tions [21]. In this section, we discuss these challenges and propose some solutions that have been considered in this field.

First, in BTTNs there is strong dependence of the range over which a tag can communicate on the distance between the tag and the source of its backscattered RF energy, whether an ambient source or an RF exciter is used. In other words, a tag which is located close to the RF energy source will be able to backscatter over a larger distance, than the same tag if it is placed further away from the RF energy source. This is, of course, different than a typical sensor/ad hoc networks, where the length of a link depends on the node itself and does not change with the location of the node. This has a number of implications in the design of the routing and MAC protocols, including the fact that the set of destination of a node in a BTTN depends on the location of the $\mathrm{RF}$ energy source relative to the node. Because of this phenomenon, there is also a larger likelihood of unidirectional links being present between two communicating tags, when the two tags are at different distances from the RF source. (In general, this likelihood depends on the tags and the distribution of RF sources.) As BTTNs tend to be distributed in their operation (i.e., there is no central element that coordinates the MAC access or the routing discovery operations) the need to perform these operations over unidirectional links is a more difficult problem than in undirected networks $([22])$, often leading to network partitions in the unidirectional graph type. Of course, preservation of network integrity is critical for most networking environments. This is unlike other typical wireless networks where each node is powered by its own battery, thus creating links with similar capabilities in the two directions, a fact that is quite often relied upon in the design of the protocols. (E.g., if node $\mathrm{A}$ sends a message to node $\mathrm{B}$, it is given that node $\mathrm{B}$ expects to be able to reply to node $\mathrm{A}$ on the link in the reverse direction.)

Second, as multiple tags are usually powered by a single RF source, any increase or decrease in the source's RF power is likely to drastically affect large portions of the network topology. Thus, movements of nodes (of the RF power sources) or changes in the RF propagation impairments of the RF sources could significantly, and more problematically nearly instantaneously, affect large portions of the network topology. Similarly, a movement of another RF source into the network coverage area would increase the lengths of at least some of the network links - and typically of all the links in a particular area thus, creating a topology with richer connectivity. This is unlike other wireless networks (e.g., typical ad hoc or sensor networks), where the changes of topology caused by a movement of a single node are mostly limited to individual nodes in the neighborhood of the moving node only. Such sizable changes of BTTN topology require a much more robust and adaptable routing approach to preserve connectivity and to maintain optimal routes. Furthermore, the fact that these changes occur with little delay, and thus little advanced warning, and the fact that these changes may occur frequently, even more exacerbate the problem.

Third, the backscattering tags experience high level of interference at various protocol layers. Interference is generally not a problem in sparse networks or networks with infrequent communications among the network nodes. But in the envisioned applications of BTTN ([21]), such as those for densely deployed IoT systems, even a simple query might cause at least some message flooding among the tags, significantly affecting the throughput of big portions of the network. This problem is further intensified in real-time IoT applications.

Fourth, in some configurations where the RF exciter is tasked with at least part of the computational functions, some part of the routing and MAC processing could be done by the exciter [20], offloading some of the complexity from the tags. On the other hand, in the case of a zero-intelligence exciter or when an ambient RF source is utilized, all the computations need to be performed distributively by the tags themselves. Thus, to adjust to different operational scenarios, the routing and MAC protocols may need to adapt to the division of processing between the tags and the exciters. Furthermore, in the case of a zero-intelligence exciter, distributed processing is especially a challenge, since the BTTN tags operate at extremely low energy levels, significantly limiting their processing capabilities. Depending on the limited processing capabilities of the tags and their extreme lowpower operation, there is a need for new approaches to design very simple MAC and routing protocols [20, 21], such as by trading the protocols' performance for processing complexity.

In applications such as IoT, the network of tags should facilitate interactions among smart objects, each tagged with a passive tag that stores information about the object, such as the object's identity, its capabilities, attributes, and past history of interactions with other objects. As an example, if the BTTN is designed to track infectious contacts among individuals, as to alert them of possible infection ([21]), the lists of contacts need to be stored and maintained in the tags. Routing among such passive tags, each being associated with a particular object, requires creation of a suitable routing infrastructure and appropriate protocols. More specifically, the routing functions consist mainly of: finding paths between specific tags or among related tags; ensuring that the communication among the tags is expedited and takes information priority into consideration; maximizing the network throughput, i.e., concurrent communications among the tags; and reducing the interference among the selected paths. Although the basic operation of the routing protocol is to facilitate communication among the tags, i.e., finding multi-hop routes among 
the tags, routing should also facilitate higher-level operations, e.g., searching for a particular object, such as other associated/related tags in the network (e.g., for all the tags that were in close contact with a tag carried by an infected individual); querying to identify all the objects with certain attributes or certain historical values, thus creating "communities of interest" among objects to facilitate interactions and information exchange among such member objects, etc.

As an example of an approach to routing in BTTN, we now briefly discuss how to address two of the specific challenges of routing in BTTN: (a) routing scalability in a densely-deployed network and (b) route discovery in the presence of unidirectional links in the network.

In a massively deployed network, such as is envisioned for IoT applications, it is difficult to discover whether a particular tag is reachable by another tag. To combat this problem, the tags can establish loose associations, creating communities of interest - a collection of related objects, which are interspersed by other objects in the network. For example, all books in a library by a particular author could be an example of a community of interest. In this way, as further explained below, rather than routing a message to a particular book (i.e., a particular tag), a message is anycasted to the "community of books by the author," rather than unicasted to a specific tag. Routing in the network is then performed based on the attributes of a community. When a node moves away or changes its attributes, it removes itself from the particular community of interest. Once a message is delivered to any member of a community of interest (i.e., anycasted), based on the attribute of the community, the member will then share the message with all the other members of its community through intra-community routes. In other words, we proposed a two-level distributed routing hierarchy, where each tag maintains a route to some members of its community of interest, so that delivery to a particular member of a community of interest requires only delivery to one (i.e., any) member of the community. The notion of communities of interest addresses a major challenge in routing in the network of tags - routing scalability. Instead of discovering routing paths between every pair of tags in the network, routing within only a much smaller community of tags is needed.

We now discuss the second challenge - discovering routing paths in unidirectional graphs. One approach to path discovery in ad hoc networks and sensor networks is through broadcasting Route Request Query (RREQ), which is a message sent from the source node to the destination node. As the RREQ propagates through the network, the nodes append their ID to the message, until the message reaches the destination. The destination extracts the accumulated route in the RREQ and creates the Route Reply Message (RREP), which is then forwarded back to the source node through reversing the accumulated route. (Any node which receives the RREQ and knows the route to the destination, can create an RREP by appending the known route to the accumulated route in the RREQ and forwarding the RREP back to the source node through reversing the accumulated route.) Unfortunately, the above process will not work in a BTTN, because many links are unidirectional only, thus reversing the route will create an infeasible path. First, we note that our route discovery operates between a source tag and a community of tags, rather than a single destination node. Second, a new RREQ/RREP process could be introduced, where a message Forward Route Request (FREQ) is broadcast by the source and propagates (with route accumulation). When the FREQ is received by any member of the community of tags, such a node now becomes the destination node. The destination node, upon receipt of the FREQ, initiates a new Backwards Route Request (BREQ), by appending the forward route from the FREQ and broadcasting the BREQ back to the source. When the BREQ arrives at the source, it now contains both, the forward and the backward routes, where the routes in the two directions are not necessarily the same. The source then creates an RREP message with the backward route and uses the forward route to send the RREP to the destination.

\section{APPLICATIONS OF BTTNS}

In this section, we first explain a fundamental operation of two tagged objects that will facilitate many applications based on object interactions, then we describe an application that involves human interactions, and finally we list a number of possible applications of BTTNs.

\subsection{Object interactions}

By object interaction we mean exchange of information between two objects with attached tags that are in the proximity of each other and that is used for some purpose. For example, tagged objects can localize themselves relative to one another or even in an absolute sense if some tagged objects serve as anchors, that is, their locations are known. Tagged objects can also track other tagged objects in their neighborhood.

The central problem here is the estimation of distances between communicating tags. One technique for distance estimation is based on multiphase backscattering, where a tag changes the phase offset of the signal that is being backscattered in a systematic manner [23]. Suppose there are two tags, Tag 1 and Tag 2, where Tag 1 acts as Tx tag with different phases. It can readily be shown that the square of the estimated amplitude of the Rx Tag 2 at the output of the envelope detector is a sinusoid that is a function of the used phase offsets and a fixed parameter that carries information about the distance between the two tags. When the roles of Tag 1 and Tag 2 are reversed, i.e., Tag 1 receives and Tag 
2 transmits, the same phenomenon occurs. Tag 1 now obtains a sinusoid but with a different fixed parameter. It turns out that when the respective parameters of the sinusoids are added, their sum is equal to $4 \pi d / \lambda$, where $d$ is the distance between the tags and $\lambda$ is the wavelength of the excitation signal. From this relationship, the distance can readily be determined.

Further, with the same line of reasoning, the tags can estimate Doppler shifts due to moving tags. Experimental results suggest that tags can estimate Doppler shifts with about the same accuracy as that obtained by active conventional RFID readers. Also, the median tracking error based on data from two tags can be as low as 2.5 $\mathrm{cm}$ [23].

\subsection{Human interactions}

An interesting application of BTTNs is related to human interactions [8]. Here we present a setting where BTTNs serve as a 'device-free' activity recognition system [8]. Namely, when the tags in the network communicate with each other, the backscatter channel state is influenced by the surrounding environment. The channel state thus carries information that can be used for classification of dynamic activities that take place in the proximity of the tags. As explained earlier, with multiphase backscattering, the communication between two tags becomes more reliable. It turns out that this is not the only advantage of the scheme. Multiphase backscattering also helps to quantify channel state information that can serve as a unique signature of activities which in turn allows for their accurate classification.

More specifically, when a Tx tag backscatters the external signal with different phases, the Rx tag can compute features of these signals. These features vary according to the dynamic alterations of the multipath wireless channel between the tags. When there is no one near the communicating tags, the amplitudes of the received signals with different phases have features that can serve as no-activity features. Similarly, when a person performs an activity near the tags, the signature of the features takes its own value and carries information about the activity. Clearly, it is important to identify good features that allow for accurate classification. For example, it has been found that the backscatter channel phase, the backscatter amplitude, and the change in excitation amplitude between two multiphase probings have a high discriminatory power for classification [8].

Experimental results suggest that with signals provided by a BTTN, one can recognize human activities with an average error of about $6 \%$. This was accomplished with 8 different activities and 9 individuals. Interestingly, this level of performance is similar to that achieved by systems that use powered, active radios. The classification results were obtained by convolutional neural networks (for details, see [8]). While the ability to recognize activities in such a fashion is already available in various other radio technologies, BTTN provides a unique approach due to its entirely batteryless operation, possibility of ubiquity and hence ability to measure a large number of tag-to-tag channels for very fine grain measurements.

\subsection{From smart cities to biomedicine}

Since the introduction of the RFID technology in the supply chain area about 15 years ago, the technical literature has provided numerous articles that promote the concept of smart homes and smart cities. One can easily imagine a smart home with BTTNs, where the tags equipped with sensors pepper the space of the home and where many of them are placed on various types of objects. The location and tracking of such objects will then readily be enabled by the functionality described in Section 5.1. Applications in smart cities include use on structures like buildings, streets, bridges, and parking spaces. The tags (with attached sensors) can be tasked to monitor air pollution, traffic, and availability of parking spaces. If the tags' density is high, these operations can be completed with high spatial resolution. The BTTNs can also be applied to perform the structural monitoring of buildings and bridges where abnormalities can be detected without actual sensing devices and instead based on the changes in the backscattered signals due to the developed abnormalities, (e.g., cracks can be found by detecting changes in distances between two tags before and after the appearance of a crack). BTTNs will also find a number of applications in medicine, environmental sensing, precision farming, and manufacturing. For more details and other applications, see a recent review on ambient backscatter communication [12].

\section{FUTURE RESEARCH DIREC- TIONS}

BTTNs offer a unique system to enable ubiquitous massively-deployed IoT. Being batteryless and small form factor, they can easily blend with everyday objects and thus almost everything can become part of the network. Current research has successfully prototyped and evaluated single BBTN links, explored their ability to characterize the intervening wireless channel (RF sensing) with applications to localization, tracking and activity recognition. Current research has also produced theoretical studies on large-scale network routing issues. But much still needs to be done to make BTTNs practical and their applications realizable. One key issue is effective power harvesting and associated power management, so that the optimal power is allocated to activities such as communication, sensing, and computation at all times. This may limit the computation needed for routing and other application level signal processing due to a limited power budget. These are trade-offs that need to be explored in very dense deployments, e.g., tags 
in a neighborhood can time multiplex various activities to achieve a network-level power management. Also, effective distributed computing techniques are needed to address processing limitation issues.

These are quite exciting times of the IoT era. The invention of tags that can form BTTNs presents a springboard for launching the concept of IoT to new heights. The possibility for connecting every tagged object in a network that turns into a part of the IoT has finally become a reality.

BTTNs offer a range of research challenges. For example, one of them is in energy harvesting and involves the design of an energy harvester with high energy efficiency over a wide input power range. Scalability and routing discovery in the presence of unidirectional links are not trivial routing tasks. Further, in security, future research should focus on balancing the security needs of BTTNs with limited resource use on the tags. Signal processing on the tags is also difficult due to the limited computing power of the tags. Future work will reveal efficient ways of processing increasing amounts of data by the tags and in a cooperative manner.

The prospects of BTTNs are quite promising, creating a driving force for their further development. The number of BTTN applications is simply staggering. In the near future, the hardware and computational aspects of the tags will continue to improve. Novel machine learning methods, possibly designed for the tags only, will be developed, and more novel techniques in the networking of the tags will be invented. With all the anticipated progress, one may argue, the BTTNs will become the true backbone of the IoT and will bring to fruition many of the benefits that have been envisioned by the IoT paradigm.

\section{CONCLUSIONS}

In this article, we presented an overview of backscatterbased communication among batteryless tags, the hardware of the tags, the scaling from a single link to a full network, and the signal processing that is carried out by the tags. Further, we listed a number of important applications with networks composed of such tags. We also discussed challenges that the tags and the network present, including challenges in energy harvesting, computing, networking, security, and distributed signal processing and decision making.

\section{ACKNOWLEDGEMENT}

The authors are thankful for the support of NSF under Awards CNS-1901182, CNS-1763843 and CNS-1763627.

\section{REFERENCES}

[1] J. Blanckenstein, J. Klaue, and H. Karl, "A survey of low-power transceivers and their applications,"
IEEE Circuits and Systems Magazine, vol. 15, no. 3, pp. 6-17, 2015.

[2] N. E. Roberts, K. Craig, A. Shrivastava, S. N. Wooters, Y. Shakhsheer, B. H. Calhoun, and D. D. Wentzloff, "A 236nw -56.5 dbm-sensitivity Bluetooth low-energy wakeup receiver with energy harvesting in 65nm CMOS," in 2016 IEEE International Solid-State Circuits Conference (ISSCC). IEEE, 2016, pp. 450-451.

[3] J. F. Ensworth and M. S. Reynolds, "BLEBackscatter: Ultralow-Power IoT Nodes Compatible With Bluetooth 4.0 Low Energy (BLE) Smartphones and Tablets," IEEE Trans. on Microwave Theory and Techniques, 2017.

[4] P. Zhang, D. Bharadia, K. Joshi, and S. Katti, "Hitchhike: Practical backscatter using commodity WiFi," in Proceedings of the 14th ACM Conference on Embedded Network Sensor Systems CDROM, ser. SenSys '16, New York, NY, USA, 2016, p. 259-271.

[5] D. Bharadia, K. R. Joshi, M. Kotaru, and S. Katti, "Backfi: High throughput WiFi backscatter," ACM SIGCOMM Computer Communication Review, vol. 45, no. 4, pp. 283-296, 2015.

[6] V. Iyer, V. Talla, B. Kellogg, S. Gollakota, and J. Smith, "Inter-technology backscatter: Towards internet connectivity for implanted devices," in Proceedings of the 2016 ACM SIGCOMM Conference, 2016, pp. 356-369.

[7] P. Zhang, C. Josephson, D. Bharadia, and S. Katti, "Freerider: Backscatter communication using commodity radios," in Proceedings of the 13th International Conference on emerging Networking EXperiments and Technologies, 2017, pp. 389-401.

[8] J. Ryoo, Y. Karimi, A. Athalye, M. Stanaćević, S. R. Das, and P. M. Djurić, "BARNET: Towards activity recognition using passive backscattering tag-to-tag network," in 16th Annual International Conference on Mobile Systems, Applications, and Services. ACM, 2018, pp. 414-427.

[9] P. V. Nikitin, S. Ramamurthy, R. Martinez, and K. Rao, "Passive tag-to-tag communication," in 2012 IEEE International Conference on RFID (RFID). IEEE, 2012, pp. 177-184.

[10] A. Y. Majid, M. Jansen, G. O. Delgado, K. S. Ytidtnm, and P. Pawetczak, "Multi-hop backscatter tag-to-tag networks," in IEEE INFOCOM 2019IEEE Conference on Computer Communications. IEEE, 2019, pp. 721-729. 
[11] V. Liu, A. Parks, V. Talla, S. Gollakota, D. Wetherall, and J. R. Smith, "Ambient backscatter: wireless communication out of thin air," in ACM SIGCOMM Computer Communication Review, vol. 43, no. 4. ACM, 2013, pp. 39-50.

[12] N. Van Huynh, D. T. Hoang, X. Lu, D. Niyato, P. Wang, and D. I. Kim, "Ambient backscatter communications: A contemporary survey," IEEE Communications Surveys \& Tutorials, vol. 20, no. 4, pp. 2889-2922, 2018.

[13] Y. Karimi, A. Athalye, S. Das, P. M. Djurić, and M. Stanaćević, "Design of backscatter-based tagto-tag system," in IEEE International Conference on RFID (RFID), 2017.

[14] C. Xu, L. Yang, and P. Zhang, "Practical backscatter communication systems for battery-free internet of things: A tutorial and survey of recent research," IEEE Signal Processing Magazine, vol. 35, no. 5, pp. 16-27, 2018.

[15] Z. J. Haas and Z. Zheng, "Waveform design for RF power transfer," U.S. Patent Application, 15959,917, 2018.

[16] J. Ryoo, J. Jian, A. Athalye, S. R. Das, and M. Stanaćević, "Design and evaluation of "BTTN": A backscattering tag-to-tag network," IEEE Internet of Things Journal, vol. 5, no. 4, pp. 2844-2855, 2018 .

[17] Z. Shen, A. Athalye, and P. M. Djurić, "Phase cancellation in backscatter-based tag-to-tag communication systems," IEEE Internet of Things Journal, vol. 3, no. 6, pp. 959-970, 2016.

[18] A. N. Parks, A. Liu, S. Gollakota, and J. R. Smith, "Turbocharging ambient backscatter communication," in ACM SIGCOMM Computer Communication Review, vol. 44, no. 4. ACM, 2014, pp. 619-630.

[19] J. Qian, A. N. Parks, J. R. Smith, F. Gao, and S. Jin, "Iot communications with $m$-psk modulated ambient backscatter: Algorithm, analysis, and implementation," IEEE Internet of Things Journal, vol. 6, no. 1, pp. 844-855, 2018.

[20] C. Liu and Z. J. Haas, "Multi-hop routing protocols for rfid systems with tag-to-tag communication," in Proceedings of the 36th IEEE Military Communications Conference. IEEE, 2017, pp. 563-568.

[21] C. Liu, Z. J. Haas, and Z. Tian, "On the design of multi-hop tag-to-tag routing protocol for largescale networks of passive tags," in IEEE Open Journal of the Communications Society. IEEE, 2020, pp. 1035-1055.
[22] P. Sinha, S. Krishnamurthy, and S. Dao, "Scalable unidirectional routing with zone routing protocol (ZRP) extensions for mobile ad-hoc networks." IEEE, Sept. 23-28, 2000, pp. 1329-1339.

[23] A. Ahmad, Y. Huang, X. Sha, A. Athalye, M. Stanaćević, S. R. Das, and P. M. Djurić, "On measuring Doppler shifts between tags in a backscattering tag-to-tag network with applications in tracking," in ICASSP 2020-2020 IEEE International Conference on Acoustics, Speech and Signal Processing (ICASSP). IEEE, 2020, pp. 9055-9059.

\section{AUTHORS}

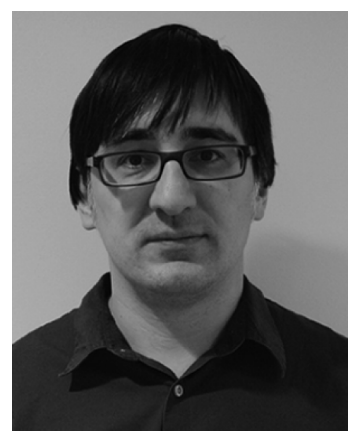

Milutin Stanaćević received the B.S. degree in Electrical Engineering from the University of Belgrade, Serbia and Ph.D. degree in Electrical and Computer Engineering from Johns Hopkins University, Baltimore, MD. He is currently an Associate Professor in the Department of Electrical and Computer Engineering at Stony Brook University, Stony Brook, NY. His research interests include mixed-signal and RF circuit and system design. Dr. Stanaćević is a recipient of the National Science Foundation CAREER award.

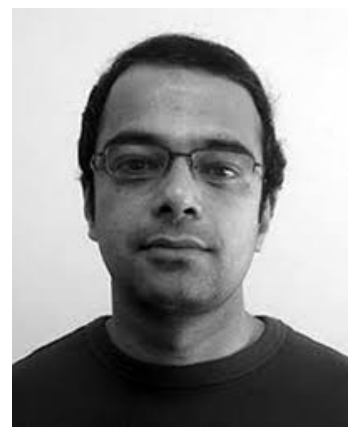

Akshay Athalye is a Co-Founder of Scandent LLC, New York, NY, USA, where he currently serves as a Chief Technology Officer. He is also an Adjunct Professor with the Department of Electrical and Computer Engineering, Stony Brook University, Stony Brook, NY, USA. He has been involved in RFID, Backscatter Communications and related research more than a decade. He has received the IEEE Region 1 Technical Excellence Award. 


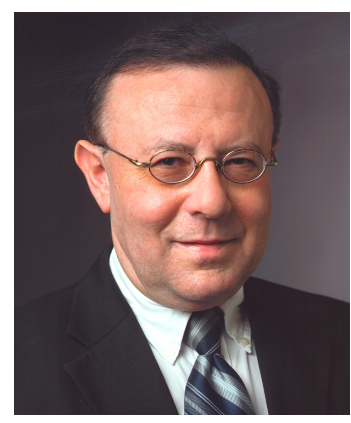

Zygmunt J. Haas has been with AT\&T Bell Laboratories from 1988 until 1995, and since 1995 with Cornell University, Ithaca, NY, USA. Since 2013, he also holds the title of a Professor and Distinguished Chair at the University of Texas at Dallas, Richardson, TX, USA. He is a recipient of numerous awards and distinctions, including IEEE Fellow, IET Fellow, EAI Fellow, and Best Paper awards, the 2012 IEEE ComSoc WTC Recognition Award, and the 2016 IEEE ComSoc AHSN Recognition Award.

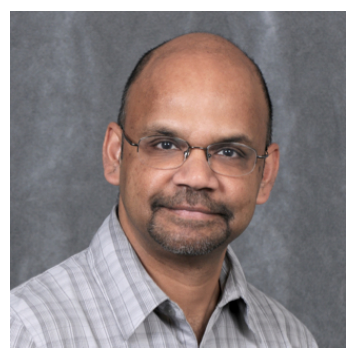

Samir R. Das is Professor in the Department of Computer Science at Stony Brook University. His research interests are in wireless networking and mobile computing, focusing on protocols, systems and performance evaluation. He co-chaired the technical program committees of premier mobile networking conferences, including ACM MobiHoc and ACM MobiCom. He served on the editorial boards of IEEE/ACM Transactions on Networking and IEEE Transactions on Mobile Computing.

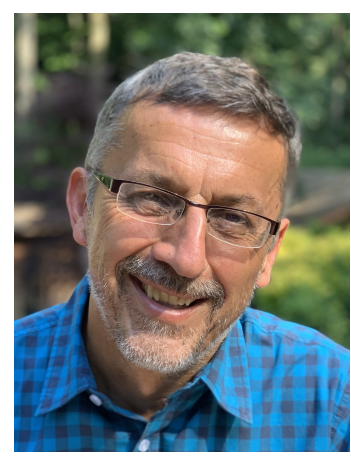

Petar M. Djurić received the B.S. and M.S. degrees in electrical engineering from the University of Belgrade, Belgrade, Yugoslavia, respectively, and the Ph.D. degree in electrical engineering from the University of Rhode Is- land, Kingston, RI, USA. He is a SUNY Distinguished Professor and currently a Chair of the Department of Electrical and Computer Engineering, Stony Brook University, Stony Brook, NY, USA. His research has been in the area of signal and information processing with primary interests in the theory of Monte Carlo-based methods; Bayesian machine learning; signal modeling, detection, and estimation; signal and information processing over networks; RFID and the IoT. Recently, his research has been applied to problems related to machine learning methods for intrapartum fetal monitoring and brain signals. He has been invited to lecture at many universities in the United States and overseas. Prof. Djurić was a recipient of the IEEE Signal Processing Magazine Best Paper Award in 2007 and the EURASIP Technical Achievement Award in 2012. In 2008, he was the Chair of Excellence of Universidad Carlos III de Madrid-Banco de Santander. From 2008 to 2009, he was a Distinguished Lecturer of the IEEE Signal Processing Society. He has been on numerous committees of the IEEE Signal Processing Society and of many professional conferences and workshops. He was Editor-in-Chief of the IEEE Transactions on Signal and Information Processing over Networks. Prof. Djurić is a Fellow of IEEE and EURASIP. 